Pacific Journal of Mathematic 


\title{
SOME MORE STATES MODELS FOR LINK INVARIANTS
}

\author{
ANDREW S. LIPSON
}

\begin{abstract}
In this paper I present two new states models for a specialization of the Kauffman link invariant $F(a, z)$ and show that these models occur naturally together. The particular specialization we are concerned with has been investigated by Lickorish and Millett and identified with a probability generating function. Kauffman has also found a description (different from the one given here) of this evaluation of $F(a, z)$.
\end{abstract}

1. The Jones polynomial $V(t)$. We begin by briefly recalling Kauffman's states model for the Jones polynomial $V(t)$, upon which the results of this paper are modelled (see [1], [3] for more details). It is interesting to compare this with the models obtained below, especially in view of the fact that $V(t)$ may be obtained from the Kauffman link invariant $F(a, z)$ of [3] by making the right substitutions for $a$ and $z$. In fact, $V(t)=F\left(t^{-3 / 4},-\left(t^{-1 / 4}+t^{1 / 4}\right)\right)$ (see [4]).

We start by defining the bracket polynomial $\langle\mathscr{D}\rangle$ of an unoriented link diagram $\mathscr{D}$. By a state $\nu$ of $\mathscr{D}$ we mean (for the moment) an assignation to each crossing $c$ in $\mathscr{D}$ of one of the two alternatives shown in Figure 1, the value $\nu(c)$ of the state at that crossing being $x$ or $x^{-1}$ accordingly.

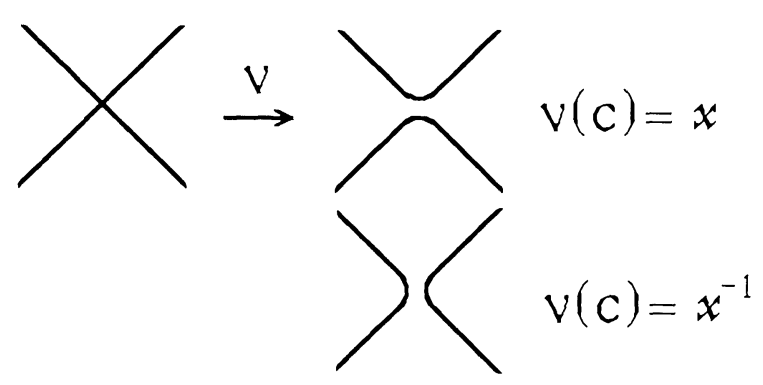

Figure 1

For a given state $\nu$, we may 'undo' each crossing in $\mathscr{D}$ in the indicated manner and then count the number of connected components of the resulting diagram $\nu(\mathscr{D})$. Write $|\nu|$ for this number. We then 


$$
\begin{aligned}
& \underline{\gamma}=x^{2} \underline{0}+\underline{0}+\int\left[+x^{-2} \Omega\right. \\
& =\int\left(+\left(x^{2}+x^{-2}-\left(x^{2}+x^{-2}\right)\right) \sim=\right)( \\
& \frac{1}{\lambda}=x^{\frac{1}{2}}+\mathrm{x}^{-1} \frac{1}{V^{\prime}}
\end{aligned}
$$

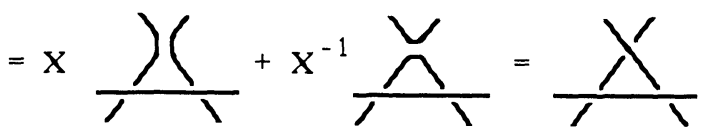

FIGURE 2

define $\langle\mathscr{D}\rangle$ by

$$
\langle\mathscr{D}\rangle=\sum_{\text {states } \nu}\left(-x^{2}-x^{-2}\right)^{|\nu|-1} \prod_{\text {crossings } c} \nu(c) .
$$

It is then easy to show that $\langle\mathscr{D}\rangle$ is invariant under the action of Reidemeister moves of types II and III (see [8]) on $\mathscr{D}$ (see Figure 2 ), and the Reidemeister move I just multiplies $\langle\mathscr{D}\rangle$ by a power of $(-x)^{3}$.

Now giving $\mathscr{D}$ an orientation, recall that the writhe $w$ of $\mathscr{D}$ is defined as the number of positive crossings minus the number of negative crossings (where these are defined as in Figure 3 ). It rapidly follows that $J(\mathscr{D})=\langle\mathscr{D}\rangle(-x)^{-3 w}$ is invariant under all three Reidemeister moves and is therefore a link invariant. Now an examination of the behaviour of $J(x)$ on skein triplets (i.e. triplets of link diagrams which are identical everywhere except in the neighbourhood of a particular crossing where they are as in Figure 3 ) leads to the discovery that it satisfies a linear relation and in fact that $J(x)=V\left(x^{4}\right)$.

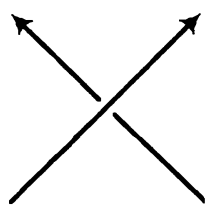

$\mathrm{L}_{+}$

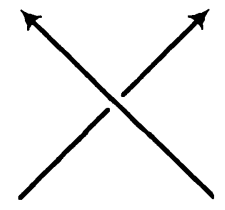

L -

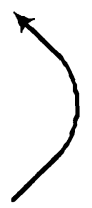

$\mathrm{L}_{\mathrm{O}}$

Figure 3 
Note that this model combines both local (what is going on at each crossing) and global (how the crossings are joined up to give distinct components in $\nu(\mathscr{D})$ ) information about $\mathscr{D}$.

2. Two new states models. We proceed in a manner very similar to that used above, first defining a polynomial [D] for each link diagram $\mathscr{D}$ in such a way that $[\mathscr{D}]$ is invariant under Reidemeister moves II and III and then multiplying by an appropriate factor to ensure invariance under Reidemeister move I as well. We first define a [.]state of $\mathscr{D}$ to be a labelling of each connected component of ( $\mathscr{D}$ crossing points) with either 1 or 2 . A [·]-state is legal if at any given crossing, each label occurs an even number of times. Each crossing must then take one of the forms illustrated in Figure 4 ( $a$ and $b$ are to be interpreted as distinct labels), and we take the value $\nu(c)$ of the state at that crossing to be as shown.

Now defining $[\mathscr{D}]$ by

$$
[\mathscr{D}]=\sum_{\text {legal states } \nu} \prod_{\text {crossings } c} \nu(c)
$$

we obtain different possibilities for states models depending on the particular values assigned to $A, B, C$ and $D$. Of course, any values give a perfectly good function on link diagrams, but in order to obtain link invariants it is necessary that the result be unchanged by the three Reidemeister moves.

Theorem 1. Let $A=B=0$ and $D=C^{-1}$. Then $R=[\cdot] C^{2}$ is a link invariant, where $w$ is the writhe. Furthermore,

$$
\frac{1}{2} R(C)=(-1)^{c(L)-1} F\left(i C^{-1}, i C-i C^{-1}\right)
$$
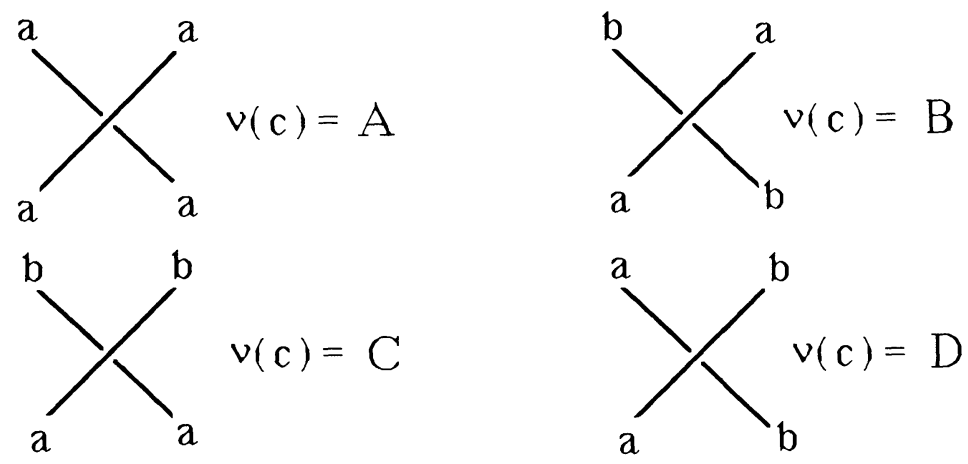

FIGURE 4 

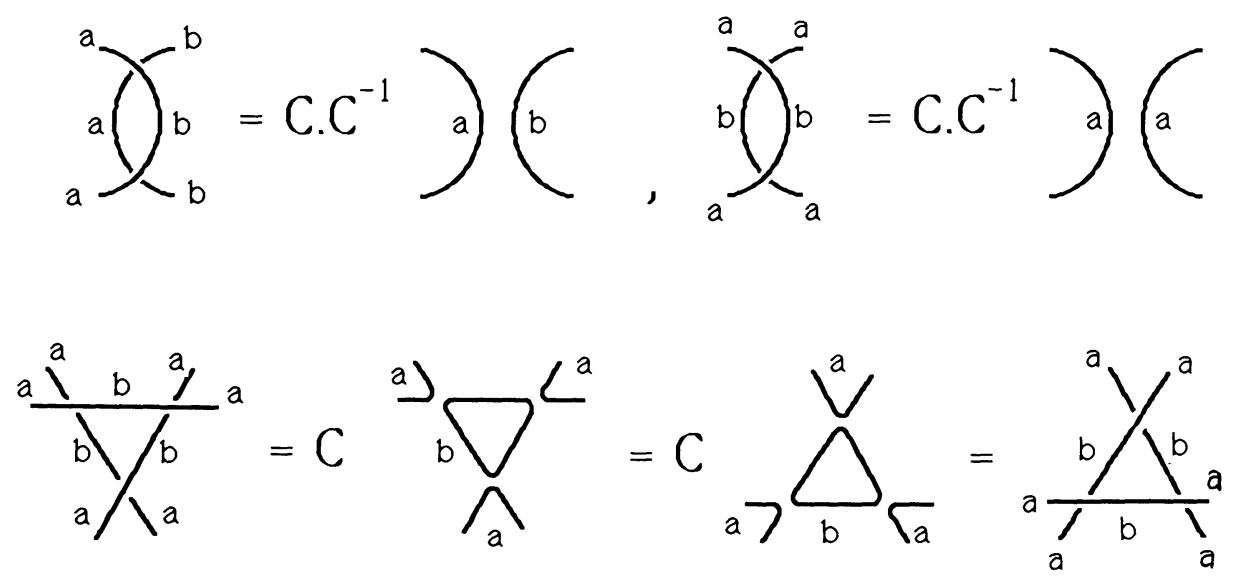

Figure 5

where $F(a, z)$ is the Kauffman link invariant and $c(L)$ is the number of components of the link $L$.

Proof. It is once again fairly easy to show that $[\mathscr{D}]$ is invariant under the second and third Reidemeister moves (see Figure 5. There are two possible labellings to consider for Reidemeister move II, both shown, and four possibilities for Reidemeister move III, of which one is shown. The reader may easily check the others).

It is convenient to allow all possible labellings of arcs of ( $\mathscr{D}$-crossing points) but to assign values of 0 to crossings of types not appearing in Figure 4 so that illegal states do not appear in the sum. Now, as before, Reidemeister move I just multiplies [ $\mathscr{D}]$ by a power of $C$, so setting $R(\mathscr{D})=[\mathscr{D}] C^{w}$ it follows that the polynomial $R$ in $C$ is a link invariant. It remains only to identify it with the claimed evaluation of $F$. Suppose given four unoriented link diagrams which are identical except in the vicinity of one particular crossing where they are as shown in Figure 6.

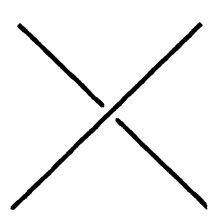

$\mathrm{L}_{+}$

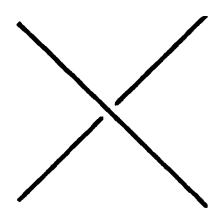

$\mathrm{L}_{-}$

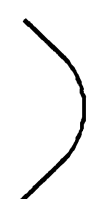

$\mathrm{L}_{\mathrm{O}}$

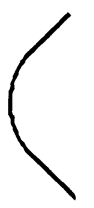

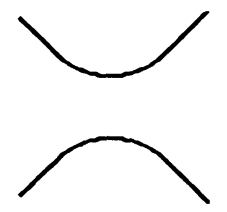

$\mathrm{L}_{\infty}$

FIGURE 6 
TABLE 1
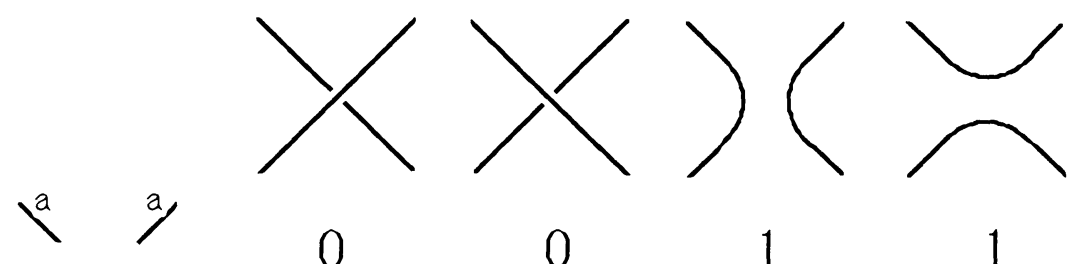

0

0

1

1

a) $\searrow^{2}$

b $b /$

C

$C^{-1}$

0

1

a) $\searrow^{2}$

a b

$C^{-1}$

C

1

0

a) $\quad 1^{b}$

Now evaluating the bracket polynomial [ $]$ on these four link diagrams we find its values are in the ratios given in Table 1 and it easily follows that

$$
[X]-[X]=\left(C^{-1}-C\right)([X]-[\cong])
$$

and we have already seen that

$$
[R]=-C^{-1}[\Omega],[R]=-C[\Lambda]
$$

This makes $R=[\cdot] C^{w}$ a case of the so-called 'Dubrovnik polynomial' $D(\alpha, \xi)$ defined by

$$
\begin{aligned}
& \Lambda(\text { K })-\Lambda(入)=\xi(\Lambda()()-\Lambda(\asymp)) \\
& \Lambda(\Omega)=\alpha \Lambda(\Lambda), \Lambda(R)=\alpha^{-i} \Lambda(\Lambda) \\
& D(\alpha, \xi)=\Lambda(\alpha, \xi) \cdot \alpha^{-w}
\end{aligned}
$$

In fact, $\frac{1}{2} R(C)=D\left(C^{-1}, C^{-1}-C\right)$. Now W. B. R. Lickorish pointed out some time ago that $D(\alpha, \xi)$ is really $F(a, z)$ in disguise via

$$
D(\alpha, \xi)=(-1)^{c(L)-1} F(i \alpha,-i \xi)
$$


where $c(L)$ is the number of components of the link $L$. Hence,

$$
R(C)=w(-1)^{c(L)-1} F\left(i C^{-1}, i C-i C^{-1}\right) .
$$

This concludes the proof of Theorem 1.

The particular evaluation of $F$ that we have encountered has already been investigated by Lickorish and Millett [6]. It is equal to

$$
\frac{1}{2}(-1)^{c(L)-1} \sum_{X \subset L} a^{-4 l k(X, L-X)}
$$

the sum being over all sublinks of the link $L$. This is the probability generating function for the linking number of a randomly-chosen sublink with its complement!

We now look at another set of values for $A, B, C$ and $D$ :

TheOREM 2. Let $A=\frac{1}{2}\left(x+x^{-1}\right), \quad B=-\frac{1}{2}\left(x+x^{-1}\right), \quad C=$ $\frac{1}{2}\left(x-x^{-1}\right)$ and $D=-\frac{1}{2}\left(x-x^{-1}\right)$. Then $s=[\cdot] x^{w}$ is a link invariant, where $w$ is the writhe. Furthermore, $S$ as a polynomial in the variable $x$ is identical to the polynomial $R$ in the variable $C$ of Theorem 1.

Proof. It is again easy to check that Reidemeister move II has no effect upon the bracket polynomial [D] of a link diagram $\mathscr{D}$, see Figure 7.

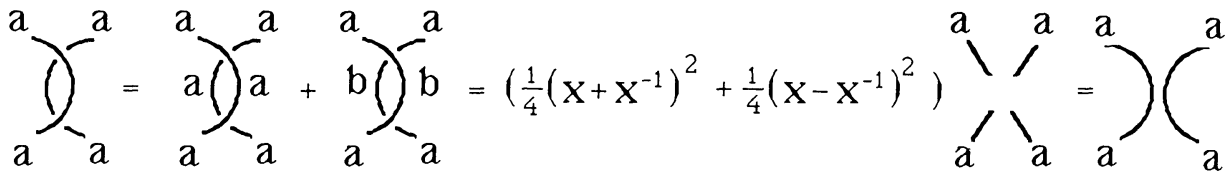

$$
\begin{aligned}
& \int_{a}^{b}=a\left(\int_{a}^{b}+b()_{a}^{b}=\frac{1}{4}\left(x+x^{-1}\right)\left(\left(x-x^{-1}\right)-\left(x-x^{-1}\right)\right)=0\right.
\end{aligned}
$$

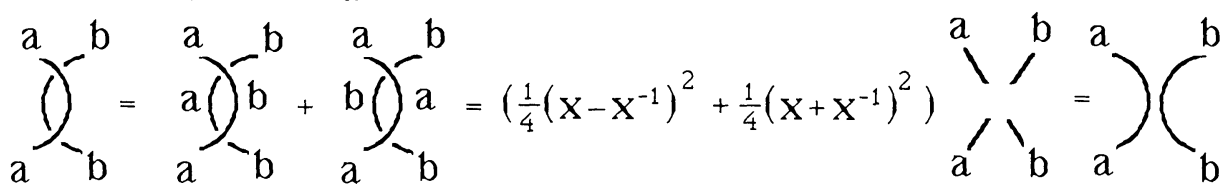

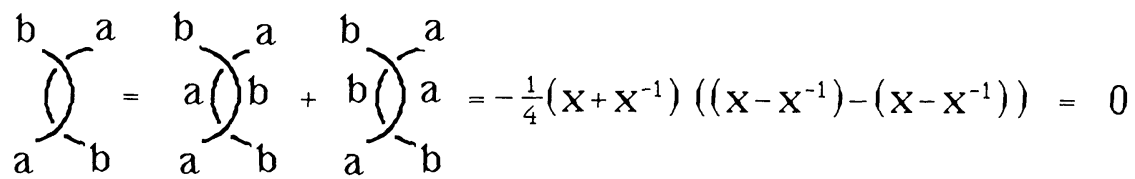


Reidemeister move III is equally easy but extremely tedious to check and adds no particular insight, so I leave it as an exercise for the computationally enthusiastic reader. As before, Reidemeister move I just multiplies $[\mathscr{D}]$ by a power of $x$, so setting $S(\mathscr{D})=[\mathscr{D}] x^{w}$ it follows that the polynomial $S$ in $x$ is a link invariant. The rest of the proof is identical to that of Theorem 1 so I will do no more than provide in Table 2 the ratios of values of $\langle\mathscr{D}\rangle$ on the four link diagrams of Figure 6.

In order to explain where these states models come from, I will briefly return to the definition of the new bracket polynomial [ $\mathscr{D}]$ in $\S 2$.

\section{TABLE 2}
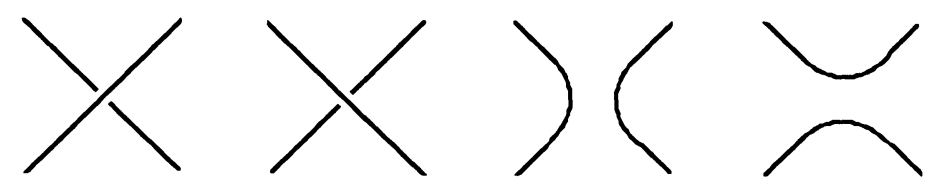
$\searrow^{a} a$
$\frac{1}{2}\left(\mathrm{X}+\mathrm{X}^{-1}\right) \quad \frac{1}{2}\left(\mathrm{X}+\mathrm{X}^{-1}\right)$
1
1
a) $\searrow^{a}$
b
$\searrow^{a} \mathrm{~b}$
b) $\backslash^{a}$
$-\frac{1}{2}\left(\mathrm{X}+\mathrm{X}^{-1}\right) \quad-\frac{1}{2}\left(\mathrm{X}+\mathrm{X}^{-1}\right)$
0
0
$1^{a} \quad a$
$\frac{1}{2}\left(\mathrm{X}-\mathrm{X}^{-1}\right) \quad \frac{1}{2}\left(\mathrm{X}-\mathrm{X}^{-1}\right)$
0
1
b $\backslash b$
b
$-\frac{1}{2}\left(\mathrm{X}-\mathrm{X}^{-1}\right)$
$-\frac{1}{2}\left(\mathrm{X}-\mathrm{X}^{-1}\right)$
1
0
a) $\searrow^{b}$ 


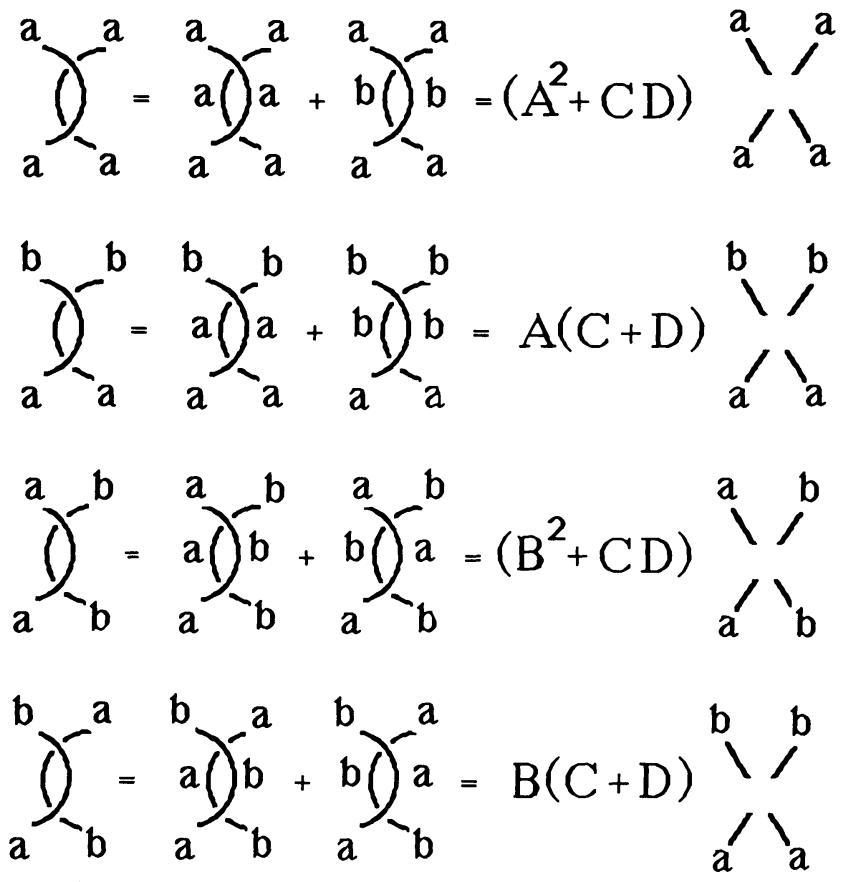

Figure 8

It is clear from Figure 8 that to obtain invariance under Reidemeister move II we are led to the relations:

$$
\begin{gathered}
A^{2}+C D=1, \\
A(C+D)=0, \\
B^{2}+C D=1, \\
B(C+D)=0 .
\end{gathered}
$$

Now there are two cases: either we may assume $A=0$ and $B=0$, in which case $C D=1$, or alternatively we have $C=-D$ and then $B^{2}=A^{2}=-C^{2}$. These two solutions lead to the states models of Theorems 1 and 2 .

I conclude this section by noting the provocative resemblance between the substitutions in $F(a, z)$ which provide the Jones polynomial, which is reducible to

$$
F\left(a^{3},-\left(a+a^{-1}\right)\right)
$$

and Lickorish/Millett's probability generating function, which is reducible to

$$
F\left(a,-\left(a+a^{-1}\right)\right)
$$


3. Special evaluations of the Jones polynomial. There are also special similar states models for particular evaluations of the Jones polynomial $V(t)$. Let $n$ be a positive integer greater than 1, and proceed as in $\S 3$ to define a bracket polynomial [.] on link diagrams. However, instead of labelling each component of ( $\mathscr{D}$-crossing points) in a diagram $\mathscr{D}$ with 1 or 2 , we allow ourselves $n$ distinct labels. Again, a legal state is one in which, at each crossing point, each label occurs an even number of times. The conditions put on $A, B, C$ and $D$ by requiring invariance under Reidemeister moves II and III are:

$$
\begin{aligned}
B & =0, \\
A & =C+C^{-1}, \\
D & =-C, \\
A^{2} & =2-n
\end{aligned}
$$

(there is another solution but it is trivial). Precisely the same arguments as used in $\S 2$ to identify $R$ show that the link invariant provided by this model is equivalent to the Jones polynomial $V\left(C^{4}\right)$. Noting that

$$
2-n=A^{2}=C^{2}+C^{-2}+2
$$

we have

THEOREM 3. Using $n$ distinct labels on the components of (D)crossing points) and setting $A=C+C^{-1}, B=0, D=-C$ and $A^{2}=2-n$, the bracket polynomial $[\mathscr{D}]$ is invariant under Reidemeister moves II and III and after renormalising with respect to the writhe provides a link invariant. Furthermore, this invariant is reducible to the Jones polynomial $V(t)$ when $t^{1 / 2}+t^{-1 / 2}=-n$.

It is interesting to note that, for small $n$, these values of $t$ seem to include some which are already known to be interesting (see, e.g. [5], [7]). Recent work by V. F. R. Jones [2] and V. G. Turaev [9] may show why these values are significant.

\section{REFERENCES}

[1] V. F. R. Jones, A polynomial invariant for knots via von Neumann algebras, Bull. Amer. Math. Soc., 12 (1985), 103-111.

[2] —_ Seminars at Cambridge, March 1987.

[3] L. H. Kauffman, States models for knot polynomials, (to appear).

[4] W. B. R. Lickorish, A relationship between link polynomials, Math. Proc. Camb. Phil. Soc., 100 (1986), 109-112. 
[5] W. B. R. Lickorish and K. C. Millett, Some evaluations of link polynomials, Comment. Math. Helvetici, 61 (1986), 349-359.

[6] _ An evaluation of the F-polynomial of a link, (to appear).

[7] A. S. Lipson, An evaluation of a lilnk polynomial, Math. Proc. Camb. Phil. Soc., 100 (1986), 361-364.

[8] K. Reidemeister, Knotentheorie, Ergebn. Math. Grenzgeb. Bd. 1; SpringerVerlag, Berlin, 1932.

[9] V. G. Turaev, The Yang-Baxter equation and invariants of links, LOMI preprint E-3-87, Leningrad 1987.

Received September 17, 1987.

Department of Pure Mathematics and Mathematical Statistics 16 Mill LANE

CAmbridge, CG2 15B, ENGland 


\title{
PACIFIC JOURNAL OF MATHEMATICS EDITORS
}

V. S. VARADARAJAN

(Managing Editor)

University of California

Los Angeles, CA 90024-1555-05

HeRbert Clemens

University of Utah

Salt Lake City, UT 84112

F. Michael Christ

University of California

Los Angeles, CA 90024-1555

THOMAS ENRIGHT

University of California, San Diego

La Jolla, CA 92093
Nicholas ERcolani

University of Arizona

Tucson, AZ 85721

R. FINN

Stanford University

Stanford, CA 94305

VAUGHAN F. R. JONES

University of California

Berkeley, CA 94720

Steven Kerckhoff

Stanford University

Stanford, CA 94305

\section{C. MOORE}

University of California

Berkeley, CA 94720

Martin ScharlemanN

University of California

Santa Barbara, CA 93106

HAROLD STARK

University of California, San Diego

La Jolla, CA 92093

\section{ASSOCIATE EDITORS}
R. Arens
E. F. BECKENBACH
B. H. NeumanN
F. WoLF
K. YoshidA
(1906-1982)
(1904-1989)

\section{SUPPORTING INSTITUTIONS}

UNIVERSITY OF ARIZONA

UNIVERSITY OF BRITISH COLUMBIA

CALIFORNIA INSTITUTE OF TECHNOLOGY

UNIVERSITY OF CALIFORNIA

MONTANA STATE UNIVERSITY

UNIVERSITY OF NEVADA, RENO

NEW MEXICO STATE UNIVERSITY

OREGON STATE UNIVERSITY

\author{
UNIVERSITY OF OREGON \\ UNIVERSITY OF SOUTHERN CALIFORNIA \\ STANFORD UNIVERSITY \\ UNIVERSITY OF HAWAII \\ UNIVERSITY OF TOKYO \\ UNIVERSITY OF UTAH \\ WASHINGTON STATE UNIVERSITY \\ UNIVERSITY OF WASHINGTON
}

The Supporting Institutions listed above contribute to the cost of publication of this Journal, but they are not owners or publishers and have no responsibility for its content or policies.

\begin{abstract}
Mathematical papers intended for publication in the Pacific Journal of Mathematics should be in typed form or offset-reproduced (not dittoed), double spaced with large margins. Please do not use built up fractions in the text of the manuscript. However, you may use them in the displayed equations. Underline Greek letters in red, German in green, and script in blue. The first paragraph must be capable of being used separately as a synopsis of the entire paper. In particular it should contain no bibliographic references. Please propose a heading for the odd numbered pages of less than 35 characters. Manuscripts, in triplicate, may be sent to any one of the editors. Please classify according to the 1991 Mathematics Subject Classification scheme which can be found in the December index volumes of Mathematical Reviews. Supply name and address of author to whom proofs should be sent. All other communications should be addressed to the managing editor, or Elaine Barth, University of California, Los Angeles, California 90024-1555-05.

There are page-charges associated with articles appearing in the Pacific Journal of Mathematics. These charges are expected to be paid by the author's University, Government Agency or Company. If the author or authors do not have access to such Institutional support these charges are waived. Single authors will receive 50 free reprints; joint authors will receive a total of 100 free reprints. Additional copies may be obtained at cost in multiples of 50 .
\end{abstract}

The Pacific Journal of Mathematics (ISSN 0030-8730) is published monthly except for July and August. Regular subscription rate: $\$ 190.00$ a year (10 issues). Special rate: $\$ 95.00$ a year to individual members of supporting institutions.

Subscriptions, orders for numbers issued in the last three calendar years, and changes of address should be sent to Pacific Journal of Mathematics, P.O. Box 969, Carmel Valley, CA 93924, U.S.A. Old back numbers obtainable from Kraus Periodicals Co., Route 100, Millwood, NY 10546.

The Pacific Journal of Mathematics at P.O. Box 969, Carmel Valley, CA 93924 (ISSN 0030-8730) is published monthly except for July and August. Second-class postage paid at Carmel Valley, California 93924, and additional mailing offices. Postmaster: send address changes to Pacific Journal of Mathematics, P.O. Box 969, Carmel Valley, CA 93924.

PUBLISHED BY PACIFIC JOURNAL OF MATHEMATICS, A NON-PROFIT CORPORATION Copyright (C) 1992 by Pacific Journal of Mathematics 


\section{Pacific Journal of Mathematics}

Vol. 152, No. $2 \quad$ February, 1992

Edoardo Ballico, On the restrictions of the tangent bundle of the

Grassmannians

Edward Burger, Homogeneous Diophantine approximation in

$S$-integers

Jan Dijkstra, Jan van Mill and Jerzy Mogilski, The space of

infinite-dimensional compacta and other topological copies of $\left(l_{f}^{2}\right)^{\omega} \ldots 255$

Mike Hoffman, Multiple harmonic series ..................... 275

Wu Hsiung Huang, Superharmonicity of curvatures for surfaces of constant

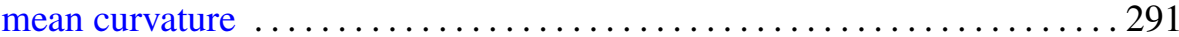

George Kempf, Pulling back bundles ......................... 319

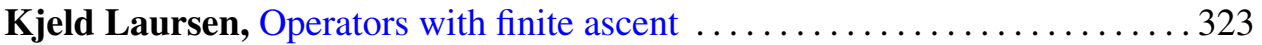

Andrew Solomon Lipson, Some more states models for link invariants . . . . 337

Xiang Yang Liu, Bloch functions of several complex variables .......... 347

Madabusi Santanam Raghunathan, A note on generators for arithmetic

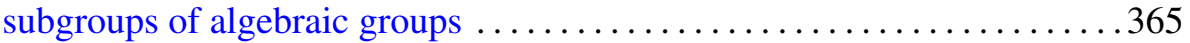

Marko Tadić, Notes on representations of non-Archimedean $\operatorname{SL}(n) \ldots \ldots 375$ 